\title{
Travail de la terre, travail du fer
}

Martial Monteil

\section{CpenEdition}

\section{Journals}

Édition électronique

URL : http://journals.openedition.org/rao/1478

DOI : 10.4000/rao.1478

ISBN : 978-2-7535-1846-9

ISSN : 1775-3732

Éditeur

Presses universitaires de Rennes

Édition imprimée

Date de publication : 31 décembre 2011

Pagination : 292-293

ISBN : 978-2-7535-1844-5

ISSN : 0767-709X

\section{Référence électronique}

Martial Monteil, «Travail de la terre, travail du fer », Revue archéologique de l'Ouest [En ligne], 28 | 2011, mis en ligne le 30 mars 2012, consulté le 06 décembre 2020. URL : http://journals.openedition.org/ rao/1478; DOI : https://doi.org/10.4000/rao.1478

Ce document a été généré automatiquement le 6 décembre 2020.

Tous droits réservés 


\title{
Travail de la terre, travail du fer
}

\author{
Martial Monteil
}

\section{RÉFÉRENCE}

Dumasy, F., Dieudonne-Glad, N., Laüt, L. et collab., 2010 - Travail de la terre, travail du fer. L'espace rural autour d'Argentomagus (Saint-Marcel, Indre), Bordeaux, Ausonius, coll.

« Mémoires ; 23 », 525 p. (ISBN 978-2-3561-3032-7).

1 Ce bel ouvrage donne à voir, de manière très détaillée, le résultat des recherches conduites depuis plusieurs décennies sur le territoire environnant l'agglomération secondaire antique d'Argentomagus, l'une des plus importantes de la cité des Bituriges Cubes. Les trois auteurs principaux ont réalisé l'essentiel des nombreux travaux qui sont utilisés ici, à titre individuel ou dans le cadre de programmes collectifs de recherche, et en proposent une synthèse qui s'inscrit dans la lignée des travaux initiés en Narbonnaise sur les réseaux de peuplement, notamment ceux qui ont été réunis dans le cadre du programme Archoomedes. L'enquête, conduite à l'échelle microrégionale, aborde de multiples problématiques dont l'intérêt est renforcé par trois originalités principales : elle offre un panorama précis, et encore rare à l'échelle des Gaules, des relations variées entre une ville secondaire et sa proche campagne; elle fournit d'abondants compléments sur la production du fer caractéristique du secteur; elle permet d'utiles comparaisons avec les thèmes similaires qui ont fait l'objet de publications récentes à l'échelle plus large de l'ensemble de la cité.

2 Le texte, partagé en quatre parties, est soutenu par une riche illustration, dont de multiples cartes qui témoignent de tout l'intérêt du traitement des données avec un système d'information géographique. Il est complété par un cd-rom qui réunit les bases de données liées à l'étude de l'atelier sidérurgique d'Oulches et de l'espace rural, utilisables sous Filemaker pro.

Dans une première partie «Les Temps de l'enquête », F. Dumasy met en place les cadres généraux de l'enquête, en insistant tout d'abord sur la production du fer qui fut l'une des principales richesses de la région, attestée par l'archéologie mais aussi par les 
sources textuelles. Elle rappelle à ce titre la mention dans la Notitia Dignitatum de l'installation aux environs d'Argentomagus, sans doute dans le courant du IV siècle, d'une Fabrica argentomagensis armorum omnium: c'est-à-dire d'une manufacture impériale de productions de toutes les armes, au contraire des huit autres fabriques signalées en Gaule qui étaient spécialisées dans un seul type d'armement. Elle dresse ensuite un bref bilan de l'histoire de la recherche et définit les limites du territoire pris en compte par confrontation avec les aires d'influence des agglomérations voisines et de même rang, soit un espace d'une vingtaine de kilomètres de rayon. Suit la présentation de l'Action thématique programmée qui a servi de socle à l'analyse et dans laquelle sont évoqués le contexte géographique et géologique ainsi que les méthodes mises en œuvre.

4 La deuxième partie " Les composantes de l'espace rural ", due à L. Laüt, est entièrement consacrée au réseau de peuplement, constitué par 172 établissement ruraux identifiés par prospection ou fouille. Les biais documentaires et les difficultés à caractériser ou à différencier certains sites sont exposés avec justesse, mais n'empêchent pas la mise en place d'une typologie en cinq grandes classes - enceintes (ou enclos), fermes en terre et bois, bâtiments ruraux maçonnés, établissements ruraux modestes ou importants. L'évolution dans le temps du système constitué par ces habitats est également abordée, révélant, comme dans bien d'autres régions des Gaules, l'importance de l'héritage laténien, l'essor du peuplement au Haut-Empire et le reflux progressif qui s'ensuit. L'analyse sitologique conduit quant à elle à noter l'absence de critère dominant dans l'implantation de ces habitats, si ce n'est la qualité et la variété des sols.

5 Les réseaux parcellaires sont également examinés à l'échelle de plusieurs petits terroirs, tandis que des traces de systèmes parcellaires de plus grande envergure sont mises en évidence, mais dont l'extension et la nature exactes restent à définir. S'y ajoutent les sites funéraires qui permettent d'établir une typologie des sépultures et débouchent sur quelques réflexions sur leur localisation et leurs relations, toujours délicates à appréhender, avec les lieux d'habitat. L. Laüt est ensuite rejointe par N. Dieudonné-Glad pour aborder la question de la sidérurgie. Dans cette région riche en minerai de fer, les traces antiques d'extraction sont difficiles à identifier, mais la documentation archéologique est abondante pour les ferriers et les ateliers de réduction, qui peuvent être hiérarchisés en fonction de leurs dimensions. L'activité de fabrication est attestée dès au moins le second âge du Fer, augmente au Haut-Empire puis paraît diminuer aux IV ${ }^{e}-V^{e}$ siècles. Dans cette partie de la cité des Bituriges Cubes, les autres types de productions sont peu attestés, si ce n'est sous la forme de carrières de pierre et de deux ateliers de tuiliers, mais dont l'importance quantitative est sans commune mesure avec celle du fer.

6 Dans la troisième partie, $\mathrm{N}$. Dieudonné-Glad présente en détail les résultats de la fouille et de l'étude de "l'atelier de réduction du Latté à Oulches", qui illustrent concrètement certains aspects abordés dans la partie précédente et les renforcent par de multiples analyses. Cet atelier, qui fonctionne principalement entre la fin du $\mathrm{III}^{\mathrm{e}}$ et la seconde moitié $\mathrm{du} \mathrm{IV}^{\mathrm{e}}$ siècle, autorise une reconstitution précise de l'organisation des structures de production et des modalités de fabrication du fer depuis l'approvisionnement jusqu'à la réalisation de produits semi-finis. Sa datation incite à y reconnaître une composante d'un réseau d'ateliers qui alimentait la Fabrica argentomagensis armorum omnium, dont l'emplacement exact n'est pas connu pour l'heure. 
7 F. Dumasy et L. Laüt reprennent ensuite la plume pour une quatrième partie sur "l'économie d'un territoire" abordant tout à tour le réseau des voies fluviales et terrestres - ces dernières étant pour une bonne part construites avec des déchets métallurgiques - et leur relation avec les établissements ruraux et les ateliers de sidérurgie. Suit une réflexion fort intéressante sur le lien entre ces deux dernières composantes de l'espace rural, qui renvoie au titre principal de l'ouvrage « travail de la terre, travail $d u$ fer ». Certains points restent évidemment à préciser, mais l'enquête montre déjà à ce stade, outre une évolution chronologique semblable, des zones de concentration différentes avec une dominante de l'agriculture au sud-est en lien avec les terres les plus fertiles et une densité plus forte de la sidérurgie au nord-est en relation probable avec le couvert forestier. Ce constat n'exclut pas pour autant l'existence de secteurs où les différentes activités sont plus imbriquées, avec des échanges probables. Les auteurs s'interrogent enfin sur les rythmes comparés du développement de la ville et de sa proche campagne.

Une solide conclusion fournit un résumé de l'ensemble des acquis, ouvre de nouvelles perspectives et rappelle que l'agglomération d'Argentomagus a tiré profit de sa situation géographique, à distance de la capitale Bourges et non loin de la frontière des peuples picton et lémovice, à un carrefour de voies de communication mais aussi à l'interface de zones offrant des ressources complémentaires (élevage, agriculture et production du fer). 